\title{
On Recognizing and Characterizing Visibility Graphs of Simple Polygons*
}

\author{
S. K. Ghosh \\ Computer Science Group, Tata Institute of Fundamental Research, \\ Homi Bhabha Road, Bombay 400 005, India \\ ghosh@tifrvax.tifr.res.in
}

\begin{abstract}
In this paper we establish four necessary conditions for recognizing visibility graphs of simple polygons and conjecture that these conditions are sufficient. We present an $O\left(n^{2}\right)$-time algorithm for testing the first and second necessary conditions and leave it open whether the third and fourth necessary conditions can be tested in polynomial time. We also show that visibility graphs of simple polygons do not possess the characteristics of a few special classes of graphs.
\end{abstract}

\section{Introduction}

Let $P=\left(p_{1}, p_{2}, \ldots, p_{n}\right)$ be a simple polygon. We say that a line segment lies inside $P$ if it does not intersect the exterior of $P$. Two points are said to be visible if the line segment joining them lies inside $P$. Note that if the line segment touches the boundary of $P$, they are still considered visible in $P$. An undirected graph $G=(V, E)$ is called the visibility graph of $P$ if the vertices $V=\left(v_{1}, v_{2}, \ldots, v_{n}\right)$ correspond to the vertices of $P$ and an edge occurs in $E$ between two vertices $v_{i}, v_{j}$ in $V$ if and only if $p_{i}$ and $p_{j}$ are visible in $P$.

* Part of this work was done when the author visited the Johns Hopkins University and was supported by NSF Grant DCR83-51468 and a grant from IBM.

Historical note: An earlier version of this paper appeared as [12] where we introduced the three necessary conditions for recognizing visibility graphs of simple polygons. Everett [8] gave a counterexample to our conjecture for sufficiency of these conditions in her Ph.D. thesis, 1990. Her counterexample essentially suggested strengthening the third necessary condition. She also suggested the stronger version of the third necessary condition, which has been proved by Srinivasaraghavan and Mukhopadhyay [21]. However, the counterexample given by Abello $e t$ al. [4] shows that even with the stronger version of the third necessary condition, the three necessary conditions are not sufficient. In this version we identify another necessary condition which also circumvents this counterexample. 
The visibility graph is a fundamental combinatorial structure in computational geometry, that is used in computing shortest paths amidst polygonal obstacles in the plane [16] and in decomposing two-dimensional shapes into clusters [19]. The problems of computing, recognizing, and characterizing visibility graphs of simple polygons have fascinated many researchers in computational geometry. Researchers have also considered the complexity of solving standard graph-theoretic problems for visibility graphs. Hershberger [15] proposed an $O(E)$-time algorithm for computing the visibility graph. Avis and Rappaport [5] proposed a polynomial-time algorithm for finding the largest clique in visibility graphs. The minimum dominating set problem in visibility graphs (known as the art gallery problem) is known to be NP-hard [17]. The maximum independent set problem in visibility graphs (known as the hidden set problem) is also known to be NP-hard [20]. However, there are polynomial-time algorithms for this problem for visibility graphs of special classes of polygons [13].

Given an undirected graph with a Hamiltonian cycle, the problem of recognizing visibility graphs is to test whether there exists a simple polygon such that:

1. The Hamiltonian cycle of the graph forms the boundary of a simple polygon.

2. Two vertices of the simple polygon are visible if and only if they correspond to adjacent vertices in the graph.

Based on the earlier version of this paper [12], Everett and Corneil [9] have proposed an algorithm for recognizing visibility graphs of spiral polygons. Since then, several results on characterization, recognition, and reconstruction of visibility graphs have appeared in the literature. For details, see [18]. However, the problem of recognizing visibility graphs in general is still an open problem.

In this paper we propose four necessary conditions for visibility graphs and prove that these conditions are sufficient to determine the convex and reflex vertices of simple polygons. We conjecture that these four necessary conditions are sufficient to recognize visibility graphs of simple polygons. We present an $O\left(n^{2}\right)$-time algorithm for testing Necessary Conditions 1 and 2 and leave it open whether Necessary Conditions 3 and 4 can be tested in polynomial time by exploiting polygonal geometry. Finally, we show that visibility graphs of simple polygons do not possess the characteristics of perfect graphs, circle graphs, or chordal graphs.

\section{Necessary Conditions}

We assume that the vertices of the given visibility graph (denoted by $V G$ ) are numbered from 1 to $n$ and the given Hamiltonian cycle is $1,2, \ldots, n$ in counterclockwise order. A cycle $i_{1}, i_{2}, \ldots, i_{k}$ in $V G$ is said to be ordered if $i_{1}, i_{2}, \ldots, i_{k}$ preserve their order in the Hamiltonian cycle. The Hamiltonian cycle is the longest ordered cycle in $V G$.

Necessary Condition 1. In a visibility graph every ordered cycle of $k \geq 4$ vertices has at least $k-3$ diagonals.

Proof. Assume on the contrary that there exists a simple polygon $P$ which contains an ordered cycle of $k$ vertices with $k-4$ diagonals or less. As the ordered cycle of $k$ vertices 


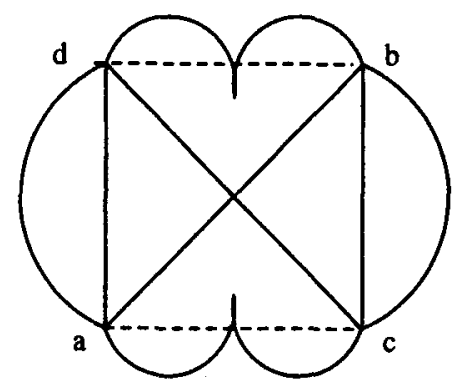

Fig. 1. The cycle $(a, b, c, d)$ is an unordered cycle in the polygon.

corresponds to a subpolygon $P^{\prime}$ and $k-3$ diagonals are necessary to triangulate $P^{\prime}, P^{\prime}$ must contain at least an ordered cycle $a, b, c, d$ without any diagonal. So, the diagonals $(a, c)$ and $(b, d)$ must intersect the exterior of the polygon. Since both diagonals intersect the exterior of the polygon, $a, b, c, d$ is an unordered cycle in the polygon, as shown in Fig. 1, which contradicts the assumption that $a, b, c, d$ is ordered.

For any two vertices $i$ and $j$ in $V G$, the vertices from $i$ to $j$, including $i$ and $j$, in clockwise and counterclockwise (i.e., Hamiltonian) order are referred to as the upper and lower chain of $(i, j)$, respectively. A pair of vertices $(i, j)$ is said to be a visible (or invisible) pair if $i$ and $j$ are adjacent (resp. not adjacent) in $V G$. Without loss of generality, we assume $i$ is always less than $j$ for an invisible pair $(i, j)$. A vertex $a$ is said to be a blocking vertex for an invisible pair $(i, j)$ if no two vertices $k$ of $\operatorname{chain}(i, a-1)$ and $m$ of chain $(a+1, j)$ are adjacent in $V G$. Vertex $a$ is called a blocking vertex because $a$ can be used to block the line of sight between $i$ and $j$ in the polygon. In Fig. 2, 6 is the blocking vertex for the invisible pair $(1,5)$ whereas the graphs in Fig. 3 is not a visibility graph of a simple polygon because the invisible pair $(3,6)$ does not have any blocking vertex. Intuitively, it is easy to see that reflex vertices in the polygon are blocking vertices and they introduce pockets in the polygonal boundary with no two pockets visible from each other as shown in Fig. 4. It has been observed by Everett [8] that all blocking vertices may not be reflex vertices in the polygon and therefore, the notion of blocking vertices is purely graph-theoretic.

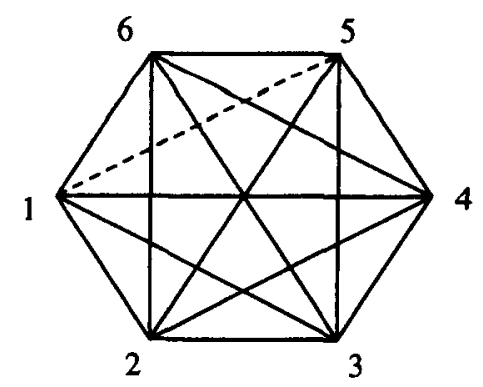

Fig. 2. Vertex 6 is the blocking vertex for the invisible pair $(1,5)$. 


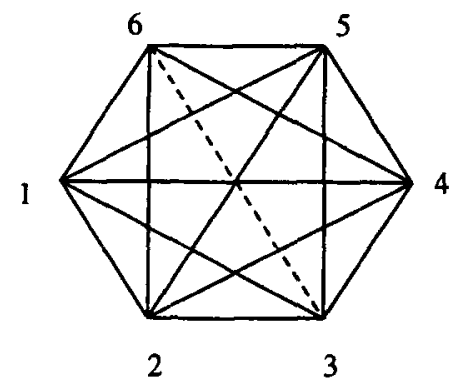

Fig. 3. The invisible pair $(3,6)$ does not have any blocking vertex.

Necessary Condition 2. Every invisible pair $(i, j)$ in a visibility graph has at least one blocking vertex.

Proof. Assume on the contrary that there exists a visibility graph of a simple polygon which does not have any blocking vertex for an invisible pair $(i, j)$. Since there is no blocking vertex for the invisible pair $(i, j)$ in the visibility graph, it implies that the Euclidean shortest path between $i$ and $j$ in the polygon does not pass through any vertex of the polygon. So, the line segment joining $i$ and $j$ lies totally inside the polygon and, therefore, $(i, j)$ is a visible pair in the visibility graph which is a contradiction.

An invisible pair can have more than one blocking vertex in either chain; critical analysis shows that such invisible pairs are redundant constraints. In the following lemmas we show that it is sufficient to consider blocking vertices for those invisible pairs which have at most one blocking vertex in each of its lower and upper chains.

Lemma 1. If $a$ and $b$ are blocking vertices in the lower chain for the invisible pair $(i, j)$, where $i<a<b<j$, then $a$ and $b$ are blocking vertices for the invisible pairs $(i, b)$ and $(a, j)$, respectively.

Proof. Since no two vertices between the chains $(i, a-1)$ and $(a+1, j)$ are visible to one another, $a$ is a blocking vertex for the invisible pair $(i, b)$. Symmetrically, $b$ is a blocking vertex for invisible pair $(i, a)$.

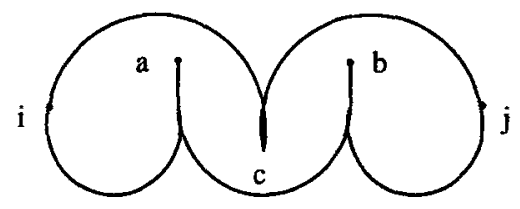

Fig. 4. No two pockets are visible from each other. 
Lemma 2. If $a$ and $b$ are blocking vertices in the lower chain for invisible pairs $(i, b)$ and $(a, j)$, respectively, where $i<a<b<j$, then $a$ and $b$ are blocking vertices for the invisible pair $(i, j)$.

Proof. Since $a$ is a blocking vertex for the invisible pair $(i, b)$, for $i \leq k \leq a-1$ and $a+1 \leq m \leq b,(k, m)$ is an invisible pair. Again, since $b$ is a blocking vertex for the invisible pair $(a, j)$, for $a \leq p \leq b-1$ and $b+1 \leq q \leq j,(p, q)$ is an invisible pair. To prove $a$ and $b$ are blocking vertices for the invisible pair $(i, j)$, it will suffice to show that there is no visible pair $(k, q)$ such that $i \leq k \leq a-1$ and $b+1 \leq q \leq j$.

Assume on the contrary that $(k, q)$ is a visible pair in $V G$ such that, for $k \leq m \leq a$ and $b \leq p \leq q,(m, p)$ is an invisible pair. Let $C$ be the smallest ordered cycle containing $k, a, b$, and $q$. Since $C$ is the smallest ordered cycle, there is no diagonal in $C$ between any two vertices of the chain $(k, a)$, between any two vertices of the chain $(a, b)$, and between any two vertices of the chain $(b, q)$. Since $a$ is a blocking vertex, there is no diagonal in $C$ between the vertices of chains $(k, a)$ and $(a, b)$. Again, since $b$ is a blocking vertex, there is no diagonal in $C$ between the vertices of the chains $(a, b)$ and $(b, q)$. Moreover, there is no diagonal between the vertices of the chains $(k, a)$ and $(b, q)$ because visible pair $(k, q)$ is chosen accordingly. Since there is no diagonal in $C$, Necessary Condition 1 is contradicted and, therefore, $(k, q)$ is an invisible pair in $V G$. So both $a$ and $b$ are blocking vertices for the invisible pair $(i, j)$.

The above lemmas correspond to the fact that some of the blocking vertices in lower or upper chains of an invisible pair form a chain of reflex vertices in the polygon as shown in Fig. 5. From now on, we only consider invisible pairs that have at most one blocking vertex in each of its lower and upper chains; call such invisible pairs minimal. An assignment is a mapping from vertices to minimal invisible pairs such that

(i) a vertex assigned to any minimal invisible pair must be its blocking vertex,

(ii) every minimal invisible pair is assigned to some blocking vertex, and

(iii) if $a$ is assigned to a minimal invisible pair $(i, j)$ where $a \in$ lower chain $(i, j)$, then

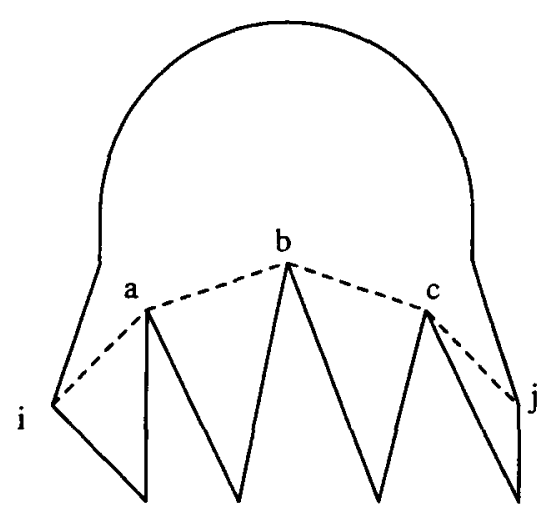

Fig. 5. Blocking vertices form a chain of reflex vertices in a polygon. 


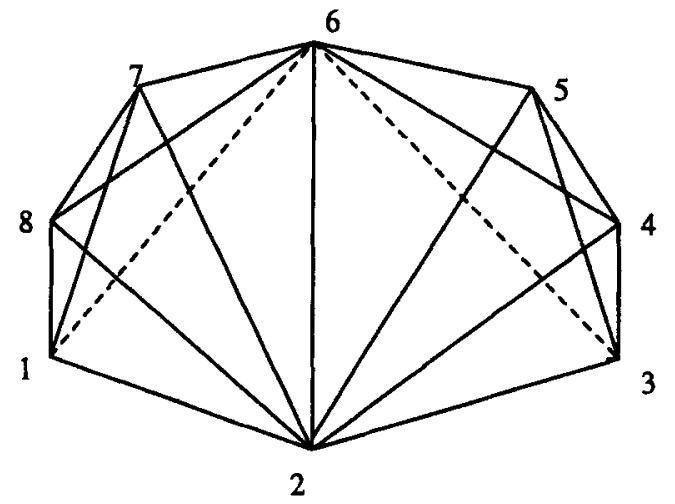

Fig. 6. Vertex 2 cannot block the visibility of separable invisible pairs $(1,6)$ and $(3,6)$ simultaneously.

$a$ is also assigned to every minimal invisible pair $(k, m)$ where $k \in \operatorname{chain}(i, a-1)$ and $m \in \operatorname{chain}(a+1, j)$.

Two invisible pairs $(i, j)$ and $(k, l)$ are said to be separable with respect to a vertex $a$ if $k$ and $l$ are encountered before $i$ and $j$ when the Hamiltonian cycle is traversed from $a$ and $a$ is the blocking vertex for both $(i, j)$ and $(k, l)$. Though it is necessary to have a blocking vertex for each invisible pair, the same blocking vertex cannot block two separable invisible pairs simultaneously. For example, 2 in Fig. 6 is the only blocking vertex for both invisible pairs $(1,6)$ and $(3,6)$ but 2 cannot block the visibility of both $(1,6)$ and $(3,6)$ as they are separable with respect to 2 . This suggests the third necessary condition.

Necessary Condition 3 [12]. Two separable invisible pairs in a visibility graph must have distinct blocking vertices.

Proof. Let $a$ be the sole blocking vertex for two separable invisible pairs $(i, j)$ and $(k, l)$ where $a$ lies in the lower chain of $(i, k)$ (Fig. 7). Since only $a$ can block the visibility between $i$ and $j$ as well as between $k$ and $l, a$ must be a reflex vertex in the polygon

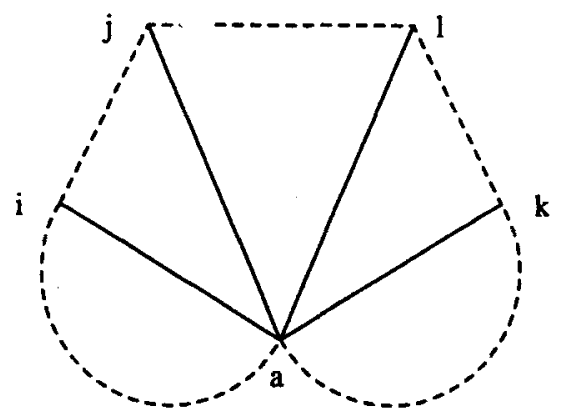

Fig. 7. Two separable invisible pairs $(i, j)$ and $(k, l)$ of a blocking vertex $a$. 


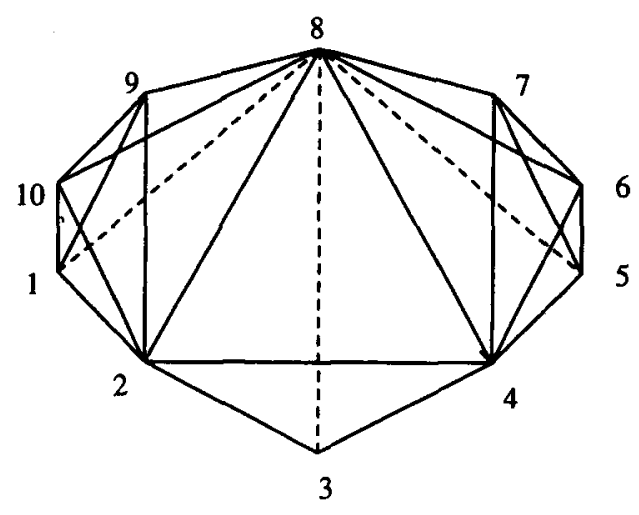

Fig. 8. This graph was proposed by Everett as a counterexample to our conjecture for sufficiency.

and lie above both $(i, j)$ and $(k, l)$. Since the interior of the two regions in the polygon bounded by the ordered cycles $i, a, j, \ldots, i$ and $a, k, \ldots, l, a$ are disjoint, $a$ cannot lie above $(i, j)$ as well as above $(k, l)$ in the polygon. Therefore $a$ cannot block the visibility between $i$ and $j$ as well as between $k$ and $l$. Hence, no two separable invisible pairs in a visibility graph can have the same blocking vertex.

In our earlier version of this paper [12], it was conjectured that these three necessary conditions are sufficient for a graph to be a visibility graph of a simple polygon. Consider the graph in Fig. 8. The graph satisfies the three necessary conditions but it is not a visibility graph since blocking vertices 2 and 4 cannot simultaneously block the visibility between invisible pairs $(1,8),(3,8)$, and $(5,8)$. This counterexample to our conjecture was proposed by Everett [8]. She also proposed the following stronger version of Necessary Condition 3, which has been proved by Srinivasaraghavan and Mukhopadhyay [21].

Necessary Condition 3 [8]. There is an assignment of vertices to all minimal invisible pairs in a visibility graph such that no blocking vertex $a$ is assigned to two or more minimal invisible pairs that are separable with respect to $a$.

Another counterexample (Fig. 9) to our conjecture of sufficiency in [12] was proposed by Abello et al. [4]. In fact this counterexample shows that the three necessary conditions are not sufficient even after strengthening Necessary Condition 3 as suggested by Everett. We now analyze why the graph in Fig. 9 is a counterexample. It can be seen that the graph satisfies Necessary Conditions 1 and 2 . Moreover, there is a unique assignment of blocking vertices to all minimal invisible pairs. The blocking vertex 1 is assigned to $(2,9)$ and $(2,10)$, the blocking vertex 2 is assigned to $(1,3)$ and $(1,4)$, the blocking vertex 5 is assigned to $(1,6),(2,6),(3,6),(4,6),(4,7)$, and $(4,8)$, and the blocking vertex 8 is assigned to $(1,7),(2,7),(5,9),(6,9),(7,9)$, and $(7,10)$. Observe that no blocking vertex is assigned to two or more of its separable invisible pairs. Therefore, this graph also satisfies even the stronger version of Necessary Condition 3. However, observe now 


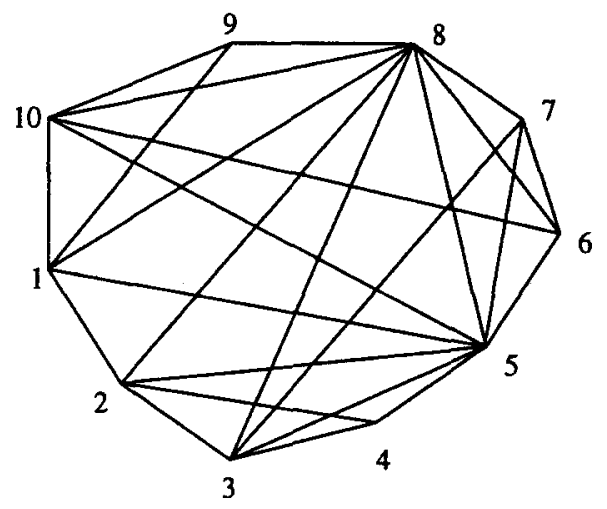

Fig. 9. This graph was proposed by Abello $e t$ al. as a counterexample to our conjecture for sufficiency.

that the graph is not a visibility graph. Note that the blocking vertices $1,2,5,8$ are reflex vertices in the subpolygon $(1,2,4,5,8,9,1)$ because $1,2,5,8$ are assigned to minimal invisible pairs $(2,9),(1,4),(4,8),(5,9)$, respectively. This implies that there are four reflex vertices in a polygon of six vertices, which is not possible. This suggests the fourth necessary condition.

Necessary Condition 4. For any assignment of blocking vertices to all minimal invisible pairs in a visibility graph, the total number of vertices of any ordered cycle $C$, which are assigned to the minimal invisible pairs between the vertices of $C$, is at most $|C|-3$.

Proof. Assume on the contrary that, for a given graph, there is an assignment and an ordered cycle $C$, such that the assignment maps $|C|-2$ or more vertices of $C$ to all minimal invisible pairs between the vertices of $C$. Let $P^{\prime}$ be the subpolygon of a simple polygon whose edges on the boundary correspond to the edges of $C$. Since each of these blocking vertices is assigned to some minimal invisible pair between the vertices of $C$, it is a reflex vertex in $P^{\prime}$. So, the sum of internal angles of $P^{\prime}$ is more than $(|C|-2) 180^{\circ}$, which contradicts the fact that the sum of internal angles of any simple polygon of $|C|$ vertices is $(|C|-2) 180^{\circ}$.

So far we have shown that any visibility graph of a simple polygon must satisfy the four necessary conditions. Moreover, we have shown in Lemmas 1 and 2 that it is sufficient to consider only minimal invisible pairs. If a minimal invisible pair $(i, j)$ has only one blocking vertex $a$, then $a$ is a reflex vertex in the polygon because only $a$ can block the line of sight between $i$ and $j$ in the polygon. If a minimal invisible pair $(i, j)$ has two blocking vertices $a$ and $b$ in opposite chains of $(i, j)$, then at least one of $a$ and $b$ must be a reflex vertex in the polygon as follows.

Let an invisible pair $(i, j)$ have exactly two blocking vertices $a$ and $b$ in the lower and upper chains of $(i, j)$, respectively (Fig. 10). Since $i, a, j$, and $b$ form an ordered cycle and $(i, j)$ is an invisible pair, $(a, b)$ must be a visible pair in $V G$ by Necessary Condition 1. Let $V_{1}=\operatorname{chain}(b+1, \ldots, i), V_{2}=\operatorname{chain}(i, \ldots, a-1), V_{3}=\operatorname{chain}(a+1, \ldots, j)$, 


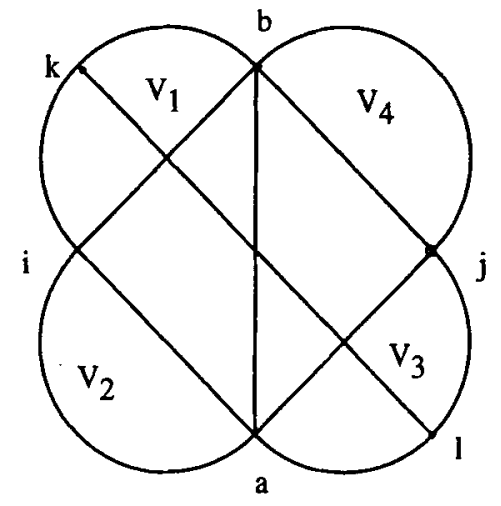

Fig. 10. Cross-visibility exists across $(a, b)$ between the vertices of $V_{1}$ and $V_{3}$.

and $V_{4}=\operatorname{chain}(j, \ldots, b-1)$. If there is a visible pair between $V_{1}$ and $V_{3}$ or $V_{2}$ and $V_{4}$, we say that there exists cross-visibility across $(a, b)$. If no cross-visibility exists across $(a, b)$, then the graph can be partitioned into two "independent" subgraphs induced by vertices $i, \ldots, a, b, \ldots, i$ and $a, \ldots, j, \ldots, b, a$. Note that every visible pair in $V G$ belongs to either of the subgraphs. So, $a$ or $b$ or both can be reflex vertices in the polygon to block the visibility between the vertices $V_{1} \cup V_{2}$ or $V_{3} \cup V_{4}$. If cross-visibility exists across $(a, b)$, then both $a$ and $b$ must be reflex vertices in the polygon and we prove it in the following theorem.

Theorem 1. Let a minimal invisible pair $(i, j)$ have exactly two blocking vertices a and $b$ in the lower and upper chains of $(i, j)$, respectively. If cross-visibility exists across $(a, b)$, then both $a$ and $b$ are reflex vertices in the polygon.

Proof. Without loss of generality, we assume that cross-visibility exists across $(a, b)$ between the vertices of $V_{1}$ and $V_{3}$. Let $(k, l)$ be a visible pair in $V G$ where $k \in V_{1}$, $l \in V_{3}, k \leq i$, and $l>a$ such that, for $i \geq m \geq k$ and $a<p \leq l,(m, p)$ is an invisible pair in $V G$ (Fig. 10). Let $C$ be the smallest ordered cycle containing $k, i, a$, and $l$. Since $C$ is the smallest ordered cycle, there is no diagonal in $C$ between any two vertices of the chain $(k, i)$ and between any two vertices of the chain $(a+1, l)$. Moreover, there is no diagonal between the vertices of the chain $(k, i)$ and the chain $(a+1, l)$ because visible pair $(k, l)$ is chosen accordingly. Therefore, $a$ is an endpoint of every diagonal in $C$. As $C$ must contain $|C|-3$ diagonals, for all $c \in C,(a, c)$ is a visible pair and, therefore, $(a, l)$ is a visible pair in $C$. Since $C$ is the smallest ordered cycle, vertices $a$ and $l$ are adjacent in $C$. Now, no vertex $m$ for $i \geq m \geq k$ in $C$ is a blocking vertex for the invisible pair $(i, l)$ because $(i, b)$ is a visible pair in $V G$. Therefore, only $a$ can block the line of sight between $i$ and $l$. Similarly, there exists an invisible pair in VG for which $b$ is the sole blocking vertex. Thus, although $a$ or $b$ can block the line of sight between $i$ and $j$, both $a$ and $b$ must be reflex vertices in the polygon. 


\section{An Algorithm for Testing Necessary Conditions}

Assume that the vertices of the given graph are numbered from 1 to $n$ according to their order in the Hamiltonian cycle, and their connectivity information is stored in an adjacency matrix. To test Necessary Condition 1, we have to check the number of diagonals in every ordered cycle. As the size of an ordered cycle varies from 4 to $n$, there are an exponential number of ordered cycles in a visibility graph and, therefore, the time complexity of the algorithm of the brute-force algorithm grows exponentially. In [12] it was left open whether Necessary Condition 1 can be tested in polynomial time by exploiting the polygonal geometry. It has been shown by Everett [8] that Necessary Condition 1 can be tested in $O\left(n^{3}\right)$ time. Here we show that Necessary Conditions 1 and 2 can be tested in $O\left(n^{2}\right)$ time.

If we consider all possible assignments of blocking vertices to minimal invisible pairs in the visibility graph, the brute-force algorithm for testing Necessary Conditions 3 and 4 runs in exponential time. Note that given an assignment, Necessary Conditions 3 and 4 can be tested in $O\left(n^{2}\right)$ time. It will be interesting to see whether Necessary Conditions 3 and 4 can be tested in polynomial time by exploiting the polygonal geometry.

Consider any three vertices $i, j$, and $k$ in Hamiltonian order. Vertices $j$ and $k$ are said to be consecutive visible vertices of $i$ if $(i, j)$ and $(i, k)$ are visible pairs and $(i, l)$ is an invisible pair for all $l \in$ chain $(j+1, k-1)$, Intuitively, $j$ and $k$ are two consecutive vertices on the boundary of the fan with $i$ as the fan vertex. In order to test Necessary Condition 1 , it is enough to check, for all $i$, whether every such pair of vertices $j$ and $k$ is connected by an edge in $V G$, as shown in the following lemmas.

Lemma 3. If the given graph $V G$ satisfies Necessary Condition 1 , then, for every vertex $i$ of $V G$, any two consecutive visible vertices of $i$ are connected by an edge in $V G$.

Proof. Assume on the contrary that $V G$ satisfies Necessary Condition 1 but there exist three vertices $i, j$, and $k$ in Hamiltonian order such that $(i, j)$ and $(i, k)$ are visible pairs and $(j, k)$ is an invisible pair. Let $C$ be the smallest ordered cycle containing $k, i, j$ and vertices of chain $(j, k)$. Since $(j, k)$ is an invisible pair by assumption and $C$ is the smallest ordered cycle, there is no diagonal between vertices of $\operatorname{chain}(j, k)$ occurring in $C$ and $|C| \geq 4$. Moreover, no vertex of $\operatorname{chain}(j+1, k-1)$ is visible from $i$ because $j$ and $k$ are two consecutive visible vertices of $i$. Hence $C$ is an ordered cycle without any diagonal, contradicting the fact that $V G$ satisfies Necessary Condition 1.

Lemma 4. If the given graph $V G$ does not satisfy Necessary Condition 1 but satisfies the condition that, for every vertex $i$ of VG, any two consecutive visible vertices of $i$ are connected by an edge in VG, then $V G$ does not satisfy Necessary Condition 2.

Proof. Since $V G$ does not satisfy Necessary Condition 1, there exists an ordered cycle $C$ of size $k$ which has less than $k-3$ diagonals. It implies that there exists another ordered cycle $C^{\prime}$ within $C$, where $\left|C^{\prime}\right| \geq 4$, such that $C^{\prime}$ does not have any diagonal. We show that there exists an invisible pair between two vertices of $C^{\prime}$ without any blocking vertex. 


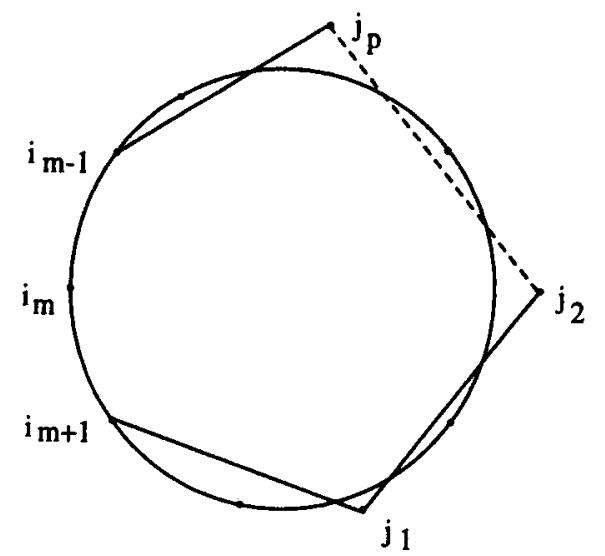

Fig. 11. The path $\left(i_{m+1}, j_{1}, j_{2}, \ldots, j_{p}, i_{m-1}\right)$ and the counterclockwise boundary of $C^{\prime}$ from $i_{m+1}$ to $i_{m+1}$ are interleaving.

Let $C^{\prime}=\left(i_{1}, i_{2}, \ldots, i_{s}\right)$. Consider any vertex $i_{m} \in C^{\prime}$. Since any two consecutive visible vertices of $i_{m}$ are connected by an edge, there is an ordered cycle $i_{m}, i_{m+1}, j_{i}, j_{2}, \ldots$, $j_{p}, i_{m-1}$ such that every vertex of this cycle is connected by an edge to $i_{m}$ (Fig. 11). Since $C^{\prime}$ is an ordered cycle without any diagonal and $\left(i_{m}, j_{1}\right),\left(i_{m}, j_{2}\right), \ldots,\left(i_{m}, j_{p}\right)$ are visible pairs, $j_{1}, j_{2}, \ldots, j_{p}$ are not vertices of $C^{\prime}$. Observe that the path $\left(i_{m+1}, j_{1}, j_{2}, \ldots, j_{p}, i_{m-1}\right)$ and the counterclockwise boundary of $C^{\prime}$ from $i_{m+1}$ to $i_{m+1}$ are interleaving. So, no vertex of $\operatorname{chain}\left(i_{m+1}, i_{m-1}\right)$ is a blocking vertex for the invisible pair $\left(i_{m+1}, i_{m-1}\right)$. It implies that $i_{m}$ is the only vertex that can block the visibility between $i_{m+1}$ and $i_{m-1}$. Since each vertex of $C^{\prime}$ is the only vertex that can block the visibility between its two adjacent vertices on $C^{\prime}$, to prove the lemma it suffices to show that there exists a vertex in $C^{\prime}$ such that it cannot block the visibility between its two adjacent vertices on $C^{\prime}$.

Let $\left(i_{m}, j_{m}\right)$ be the visible pair such that $i_{m+1}$ and $j_{m}$ are consecutive visible vertices of $i_{m}$, where $j_{m} \in \operatorname{chain}\left(i_{m+1}, i_{m-1}\right)$ (Fig. 12). If $j_{m} \in \operatorname{chain}\left(i_{m+1}, i_{m+2}\right)$, then $i_{m+1}$ cannot block the invisible pair $\left(i_{m}, i_{m+2}\right)$. So, we assume that $j_{m}$ does not belong to chain $\left(i_{m+1}, i_{m+2}\right)$ and it belongs to chain $\left(i_{t}, i_{t+1}\right)$ for some $t$. Again, let $\left(i_{m+1}, j_{m+1}\right)$ be the visible pair such that $i_{m+2}$ and $j_{m+1}$ are consecutive visible vertices of $i_{m+1}$. So, $j_{m+1}$ must belong to chain $\left(i_{m+2}, j_{m}\right)$ because $\left(i_{m+1}, j_{m}\right)$ is a visible pair. Further, $j_{m+1}$ does not belong to chain $\left(i_{m+2}, i_{m+3}\right)$ because then $i_{m+2}$ cannot block the invisible pair $\left(i_{m+1}, i_{m+3}\right)$. So $j_{m+1}$ must belong to chain $\left(i_{m+3}, j_{m}\right)$. This argument can be repeated to claim that $j_{m+1}(l \geq 1)$ must lie on $\operatorname{chain}\left(i_{m+l+2}, j_{m+l-1}\right)$. Observe that by this repetition we arrive at a vertex $i_{m+l+1}$ such that $j_{m+l+1}$ lies in $\operatorname{chain}\left(i_{m+l+2}, j_{m+l}\right)$. Hence $i_{m+l+2}$ cannot block the visibility between $i_{m+l+1}$ and $i_{m+l+3}$.

The above lemmas suggest that, for testing Necessary Condition 1, it is enough to check whether any two consecutive visible vertices of every vertex are connected by an edge in $V G$. The time required to check the visible vertices of a fixed vertex $i$ is proportional to the number of visible vertices of $i$. So the time required to check for all vertices is proportional to the total number of visible pairs in $V G$, which is $O\left(n^{2}\right)$. 


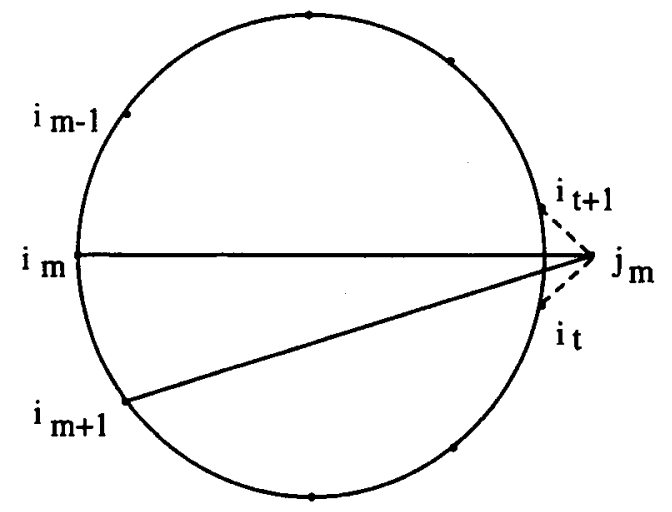

Fig. 12. Vertices $i_{m+1}$ and $j_{m}$ are consecutive visible vertices of $i_{m}$.

By the definition of a blocking vertex, Necessary Condition 2 can be tested by checking for every invisible pair $(i, j)$ that there exists a vertex $a$ in the lower (or upper) chain of $(i, j)$ such that no two vertices between chain $(i, a-1)$ (resp. chain $(a+1, i))$ and chain $(a+1, j)$ (resp. chain $(j, a-1)$ ) are adjacent in $V G$. Since the number of invisible pairs in $V G$ is $O\left(n^{2}\right)$, this naive method takes $O\left(n^{4}\right)$ time. In the following we show that Necessary Condition 2 can be tested in $O\left(n^{2}\right)$ time.

Instead of finding a blocking vertex for every invisible pair, our procedure for every vertex $i$ of $V G$ locates those invisible pairs that can be blocked by $i$. If there exists an invisible pair that cannot be blocked by any vertex, then $V G$ does not satisfy Necessary Condition 2.

We now state the procedure for locating those invisible pairs that can be blocked by $i$. Observe that if $i$ is a blocking vertex for both invisible pairs $(j, q)$ and $\left(j, q^{\prime}\right)$ where $q^{\prime} \in \operatorname{chain}(q, j)$, then $i$ is a blocking vertex for all invisible pairs $(j, l)$ where $l \in \operatorname{chain}\left(q, q^{\prime}\right)$. This observation suggests that if $(i+1, k)$ is the visible pair such that no vertex of chain $(k+1, i-1)$ is adjacent to $i+1$ in $V G$, then $i$ is a blocking vertex for all invisible pairs $(i+1, l)$ where $l \in \operatorname{chain}(k+1, i-1)$ (Fig. 13). Note that if $k=i-1$, then $i$ is not a blocking vertex for any invisible pair in $V G$. Also note that if $i$ is a blocking vertex for any invisible pair in $V G$, then one of the vertices of such an invisible pair must belong to chain $(k+1, i-1)$. So, a variable range is maintained which is initialized by $k$. Similarly, let $(i+2, k)$ be the visible pair such that no vertex of $\operatorname{chain}(k, i-1)$ is adjacent to $i+2$ in $V G$. If $k$ belongs of chain (range, $i-1$ ), assign $k$ to range. So, $i$ is a blocking vertex for all invisible pairs $(i+2, l)$ where $l \in$ chain(range $+1, i-1)$. Thus by traversing the Hamiltonian cycle in counterclockwise order from $i+1$ to $i-3$, all invisible pairs can be located such that $i$ is one of their blocking vertices. Note that after some iterations if range becomes $i-1$, then $i$ cannot block any more invisible pairs.

For locating invisible pairs where $j$ is one of their vertices and $i$ is their blocking vertex, the visible pair $(j, k)$ is located such that no vertex of chain $(k, i-1)$ is adjacent to $j$ in $V G$. To locate such a visible pair $(j, k)$ in $O(1)$ time, a circular list is maintained for each vertex $j$ of $V G$. The circular list for $j$ consists of all pairs $(j, j+1),(j, j+$ $2), \ldots,(j, j-1)$ and each pair has a pointer indicating its next clockwise visible pair in 


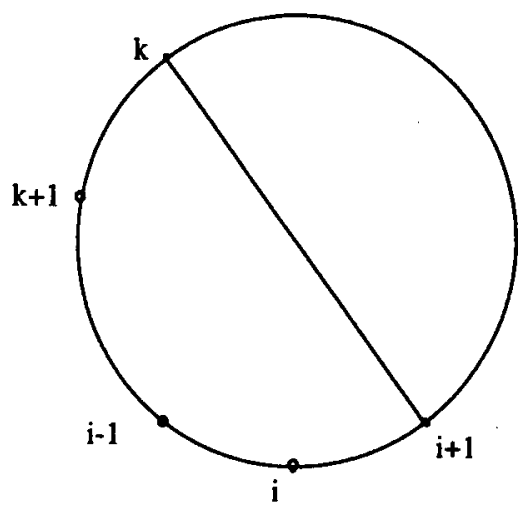

Fig. 13. No vertex of $\operatorname{chain}(k+1, i-1)$ is adjacent to $i+1$ in $V G$.

the list. In Fig. 14, $(5,7)$ is the next clockwise visible pair of the invisible pair $(5,1)$ in the circular list for vertex 5. Moreover, to locate any pair in the list in $O(1)$ time, there is a pointer in each location of the adjacency matrix to its corresponding pair in the list. Since the number of such pairs $(j, k)$ located by the algorithm can be $O\left(n^{2}\right)$ in the worst case, the total time required to locate all of them is $O\left(n^{2}\right)$.

If $i$ is found to be a blocking vertex for an invisible pair $(j, q)$ and $(j, q)$ already has a blocking vertex $a$ such that both $a$ and $i$ belong to the upper or lower chain of $(j, q)$, then $(j, q)$ is not a minimal invisible pair. Therefore $(j, q)$ is deleted in $O(1)$ time from circular lists of $j$ and $q$ and the pair is not considered subsequently by the procedure. Since the procedure locates at most two blocking vertices for any minimal invisible pair and at most three blocking vertices for other invisible pairs, the time required is proportional to the number of invisible pairs, which is $O\left(n^{2}\right)$. Since circular lists for all vertices can be initialized in $O\left(n^{2}\right)$ time and subsequent operations require $O\left(n^{2}\right)$ time, the overall time required to test Necessary Condition 2 is $O\left(n^{2}\right)$. We summarize the result in the following theorem.

Theorem 2. Given a graph of $n$ vertices, Necessary Conditions 1 and 2 can be tested in $O\left(n^{2}\right)$ time.

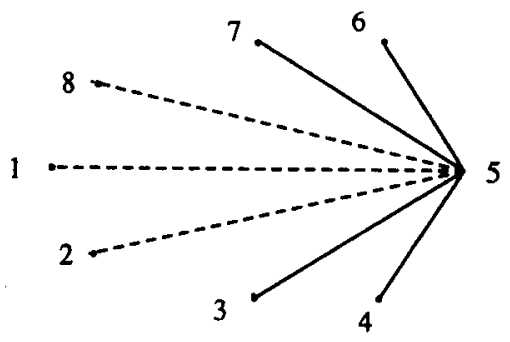

Fig. 14. The circular list for vertex 5 . 


\section{Characterization Problem}

Previous discussions show that visibility graphs of simple polygon have natural structures and characterizing these structures may turn out to be useful in designing efficient algorithms for geometric problems. From Necessary Condition 1, it seems that visibility graphs of simple polygons may fall into one of the known special classes of graphs, e.g., perfect graphs, circle graphs, or chordal graphs, but we have found counterexamples in all cases even for special classes of polygons. For an excellent survey of special classes of graphs, refer to [14].

An undirected graph $G$ is called a perfect graph [14] if $X(A)=w(A)$ for every induced subgraph $A$ of $G$ (including $G$ itself). Here $w(G)$ denotes the maximum cardinality clique of $G$ and $X(G)$ is the chromatic number of $G$. Vertices $2,8,4,10$, and 6 in the visibility graph of a simple polygon in Fig. 15 form an odd cycle without a diagonal and, therefore, the chromatic number is not equal to the maximum cardinality clique. Even in the case of a convex fan, the visibility graph is not a perfect graph. Vertices 2,6, 5,4 , and 8 form an odd cycle without a diagonal in the visibility graph of a convex fan in Fig. 16.

An undirected graph $G$ is called a circle graph [10] if there exists a set of chords $C$ on a circle and one-to-one correspondence between vertices of $G$ and chords of $C$ such that two distinct vertices are adjacent in $G$ if an only if their corresponding chords intersect. A graph $G$ is not a circle graph if $G$ contains a wheel (Fig. 17) as its subgraph [6]. In Fig. 16, vertex 1 and the cycle $2,6,5,4$, and 8 have formed a wheel. This counterexample also shows that visibility graphs do not belong to the union of perfect graphs and circle graphs.

An undirected graph $G$ is called a chordal graph [11] if every cycle with four or more vertices has a diagonal. Since chordal graphs are perfect graphs, visibility graphs of simple polygons are not chordal graphs. Even in the case of a staircase polygon, the visibility graph is not a chordal graph. In Fig. 18, vertices $16,10,12$, and 6 form a cycle without a diagonal.

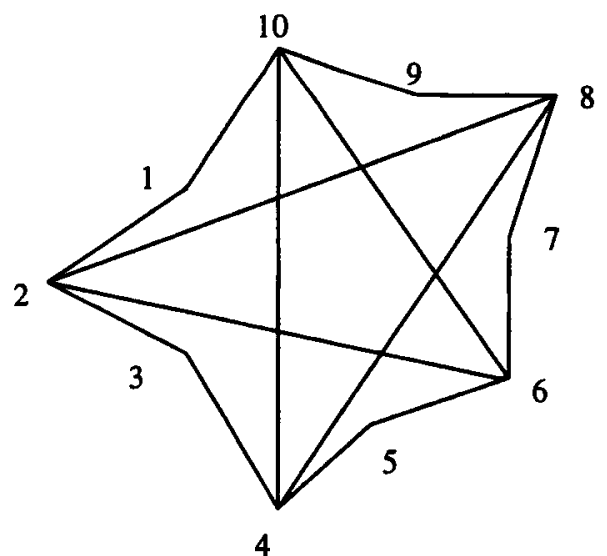

Fig. 15. Vertices $2,8,4,10$, and 6 form an odd cycle without a diagonal. 


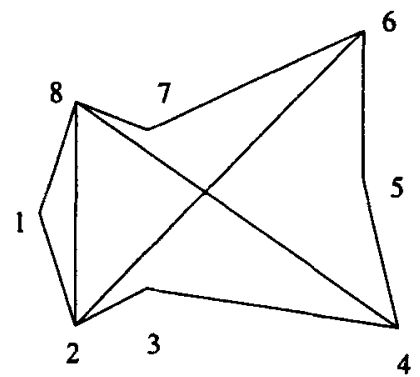

Fig. 16. Vertices $2,6,5,4$, and 8 form an odd cycle without a diagonal.

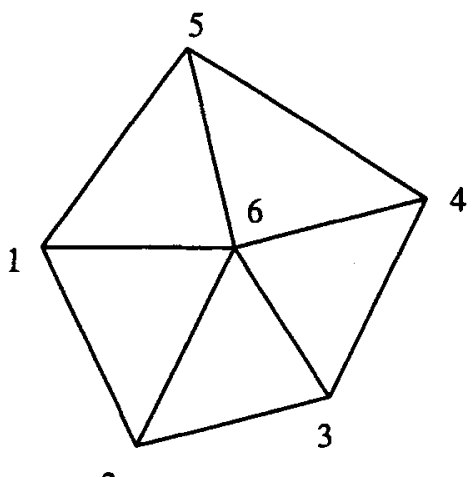

2

Fig. 17. Vertices $1,2,3,4,5$, and 6 form a wheel.

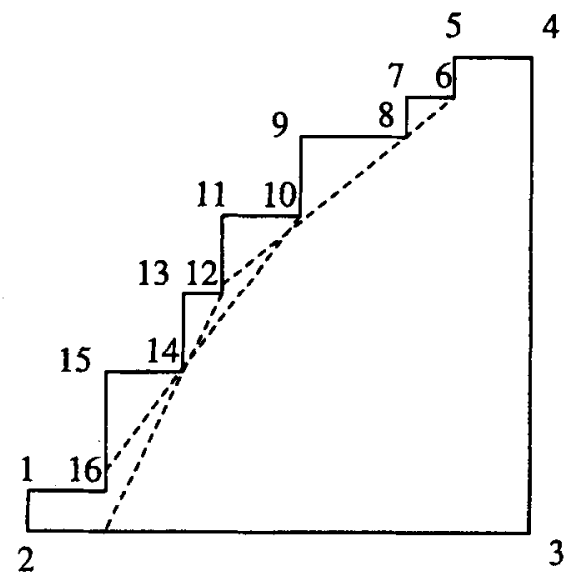

Fig. 18. Vertices 16, 10,12, and 6 form a cycle without a diagonal. 
The counterexamples demonstrate that visibility graphs of simple polygons are not highly structured graphs but the possibility of characterizing visibility graphs in terms of any known class of graphs cannot be totally ruled out. So far, visibility graphs of special classes of polygons such as spiral polygons [9], staircase polygons [1], and convex fans [2], [7] have been characterized. However, we feel that visibility graphs of simple polygons in general form a new class of graphs.

\section{Concluding Remarks}

We have established four necessary conditions for recognizing visibility graphs of simple polygons and have identified which vertices must be reflex and which vertices might be reflex in the polygon. To show that these four conditions are sufficient, we have to establish that whenever a given graph satisfies the four conditions, there exists a polygon which satisfies the visibility constraints of the given graph.

Suppose we want to reconstruct the polygon from the graph in Fig. 19. The invisible pairs are $(1,6),(1,9),(1,10),(2,5),(2,6),(2,7),(2,10),(3,5),(3,6),(3,7),(3,8),(3,9)$, $(3,10),(3,11),(4,6),(4,7),(4,10),(5,13),(6,12),(6,13),(6,14),(7,13),(8,13),(9,12)$, $(9,13),(9,14),(10,12),(10,13),(10,14),(11,13),(11,14)$. The blocking vertices are $4,5,11$, and 12 and they are visible to one another. Now we place each blocking vertex at the corner of a square and draw lines through them as shown in Fig. 20. Observe that lines drawn through blocking vertices divide the plane into regions. The next vertex $i$ added to the configuration is placed in a region which satisfies the visibility constraints of $i$. Thus, at each iteration, we add a vertex $i$ to the configuration and draw lines from $i$ through the appropriate blocking vertices. However, at some iteration, we may not find any region which satisfies the visibility constraints of $i$. For example, it can be seen that

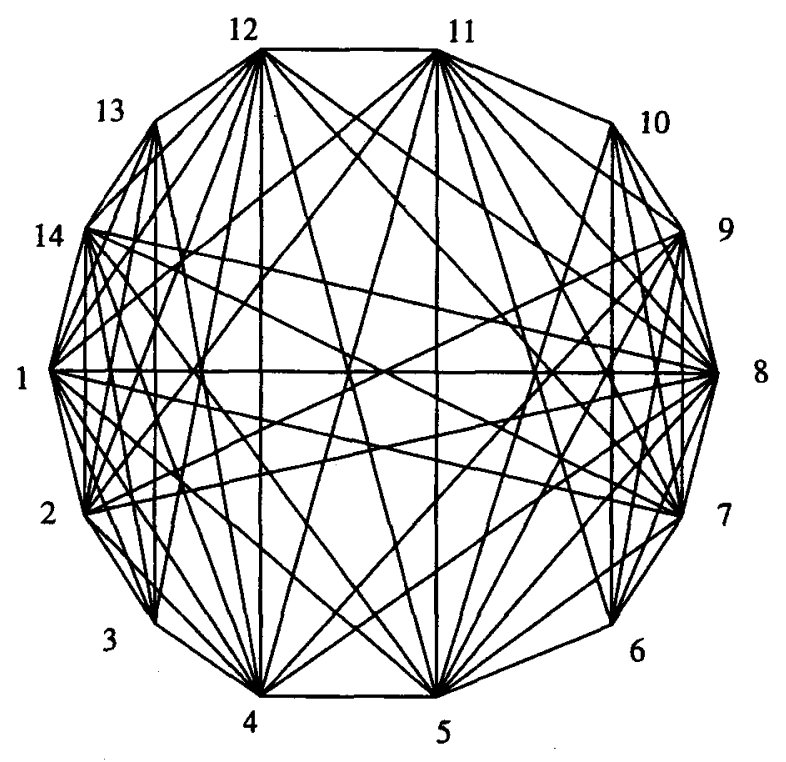

Fig. 19. A given graph. 


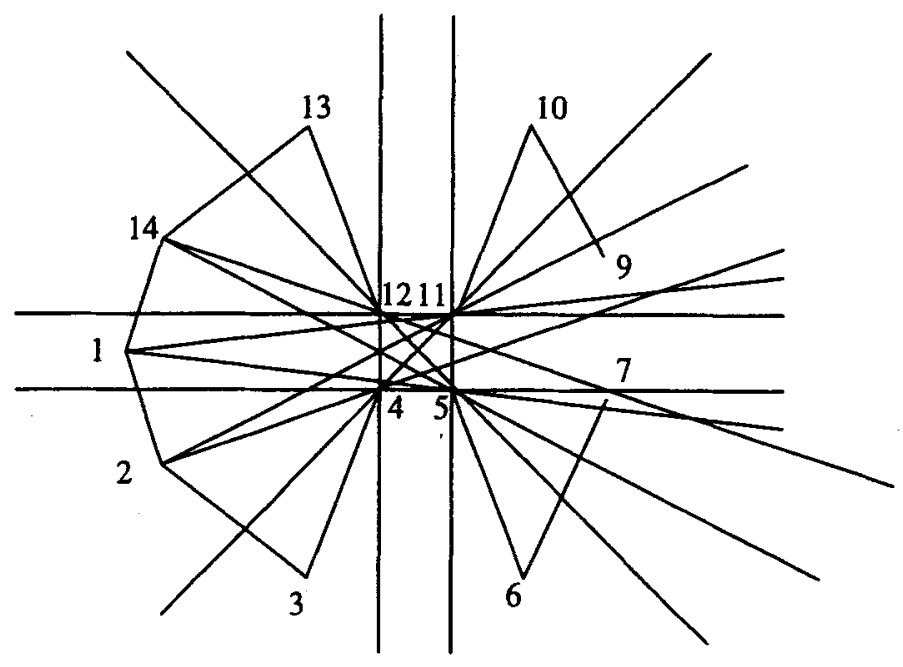

Fig. 20. Constructing a simple polygon from the graph.

vertex 8 cannot be added to the configuration since there is no region which satisfies the visibility constraints of vertex 8 . In such a situation we have to rearrange the positions of existing vertices so that an appropriate region is created for $i$. Though it seems that such a rearrangement can always be found whenever the four necessary conditions are satisfied, it is not entirely clear from a topological point of view how to settle this issue. So, we conclude with the conjecture that the four necessary conditions are sufficient to recognize visibility graphs of simple polygons.

As pointed out in the Introduction, several results on the problem of recognizing visibility graphs of various classes of graphs have appeared in the literature. Recently, Abello and Kumar [3] have independently suggested four new necessary conditions for recognizing visibility graphs of simple polygons, in addition to the three necessary conditions proposed in the first version of this paper [12]. It can be easily seen that the first necessary condition of Abello and Kumar, which they call locally inseparable, follows from Necessary Condition 3 and the definition of the assignment of blocking vertices. In the following we show that the second, third, and fourth necessary conditions proposed by Abello and Kumar, which correspond to the properties of the Euclidean shortest path between any two vertices in a polygon, follow from Necessary Condition 4.

Let $\operatorname{ESP}\left(i_{1}, i_{j}\right)=\left(i_{1}, i_{2}, \ldots, i_{j}\right)$ denote the Euclidean shortest path from a vertex $i_{1}$ to another vertex $i_{j}$ of a polygon. It is known that $\operatorname{ESP}\left(i_{j}, i_{1}\right)=\left(i_{j}, i_{j-1}, \ldots, i_{1}\right)$, i.e., two paths are identical. So, any assignment of blocking vertices to minimal invisible pairs must ensure that this property of the Euclidean shortest path between any two vertices in the corresponding polygon is preserved. Abello and Kumar have suggested this property, which they call path-symmetric, as the second necessary condition. We show that the second necessary condition of Abello and Kumar follows from Necessary Condition 4.

Assume on the contrary that there exists an assignment of blocking vertices which satisfies Necessary Condition 4 but does not satisfy the second necessary condition of 


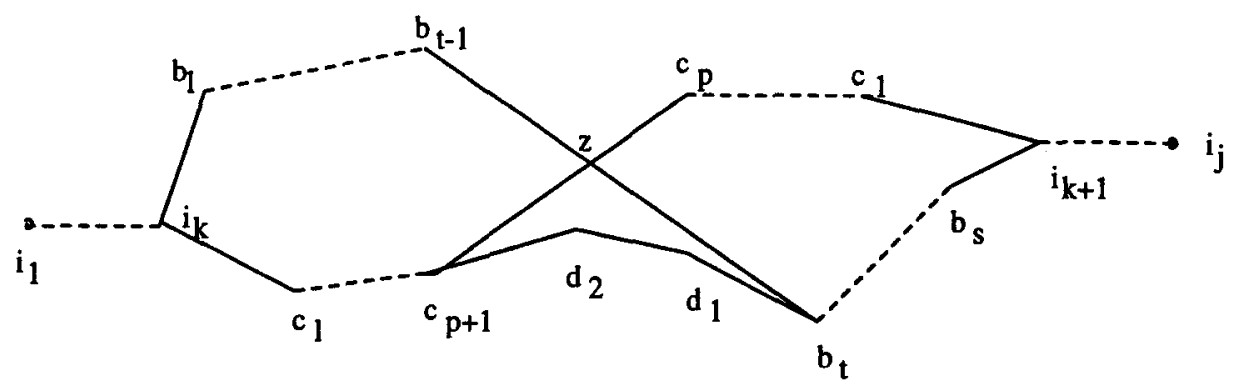

Fig. 21. Two paths between vertices $i_{1}$ and $i_{j}$.

Abello and Kumar for paths between two vertices $i_{1}$ and $i_{j}$ (Fig. 21). Let $\left(i_{1}, i_{2}, \ldots, i_{k}\right.$, $b_{1}, b_{2}, \ldots, b_{s}, i_{k+1}, \ldots, i_{j}$ ) be the path in the graph from $i_{1}$ to $i_{j}$ such that every vertex (except $i_{1}$ and $i_{j}$ ) in the path is the blocking vertex assigned to the minimal invisible pair formed by its adjacent two vertices on the path. Analogously, let $\left(i_{j}, \ldots, i_{k+1}, c_{1}, c_{2}, \ldots\right.$, $c_{l}, i_{k}, \ldots, i_{1}$ ) be the path in the graph from $i_{j}$ to $i_{1}$ such that every vertex except $i_{j}$ and $i_{1}$ in the path is the blocking vertex assigned to the minimal invisible pair formed by its adjacent two vertices on the path. Since these two paths are not identical by assumption, there exists two subpaths $\left(i_{k}, b_{1}, b_{2}, \ldots, b_{s}, i_{k+1}\right)$ and $\left(i_{k+1}, c_{1}, c_{2}, \ldots, c_{l}, i_{k}\right)$ which form a cycle $C$ in the graph and all vertices in $C$ are distinct. If $C$ is an ordered cycle, then all vertices except $i_{k}$ and $i_{k+1}$ are reflex in the corresponding subpolygon, which contradicts the assumption that the assignment of blocking vertices has satisfied Necessary Condition 4 . So, we assume that $C$ is an unordered cycle. Let $P^{\prime}$ be the crossing subpolygon corresponding to $C$. Let $z$ be the first intersection point encountered while traversing $P^{\prime}$ from $i_{k}$. Let $\left(c_{p}, c_{p+1}\right)$ and $\left(b_{t-1}, b_{t}\right)$ be the visible pairs such that their corresponding edges in $P^{\prime}$ have intersected at $z$ (Fig. 21). So, there exists another ordered cycle $C^{\prime}$ consisting of $i_{k}, b_{1}, b_{2}, \ldots, b_{l}, d_{1}, d_{2}, \ldots, c_{p+1}, \ldots, c_{l}, i_{k}$ such that every vertex of $C^{\prime}$ except $i_{k}$ and $b_{t}$ is a blocking vertex assigned to the minimal invisible pair formed by its adjacent vertices on $C^{\prime}$. Since all vertices except $i_{k}$ and $b_{t}$ are reflex in the corresponding subpolygon of $C^{\prime}$, the assignment of blocking vertices cannot satisfy Necessary Condition 4 , which is a contradiction.

We now show that the third necessary condition of Abello and Kumar also follows form Necessary Condition 4. It is known that if $\operatorname{ESP}(i, k)$ passes through vertex $j$ and $\operatorname{ESP}(j, l)$ passes through vertex $k$, then $\operatorname{ESP}(i, l)$ passes through both $j$ and $k$. So, any assignment of blocking vertices to minimal invisible pairs must ensure that this property of the Euclidean shortest path between any two vertices in the corresponding polygon is preserved. Abello and Kumar have suggested this property, which they call the first path-consistent assignment, as the third necessary condition. Assume on the contrary that there exists an assignment of blocking vertices which satisfies Necessary Condition 4 but does not satisfy the third necessary condition of Abello and Kumar. Using analogous arguments to those mentioned above, it can be shown that there exists an ordered cycle $C$ formed by the two subpaths between $j$ and $k$ such that all vertices of $C$ except $j$ and $k$ are assigned to minimal invisible pairs between the vertices of $C$, which contradicts that the assignment of blocking vertices has satisfied Necessary Condition 4. 
We now show that the fourth necessary condition of Abello and Kumar also follows from Necessary Condition 4 . Assume that $\operatorname{ESP}(i, j)$ passes through a vertex $a$. If $a \in$ lower chain $(i, j)$, then, for any two vertices $k$ and $l$ where $k \in$ chain $(i, a-1)$ and $l \in$ $\operatorname{chain}(a+1, j), \operatorname{ESP}(k, l)$ also passes through $a$. Analogously, if $a \in \operatorname{upper} \operatorname{chain}(i, j)$, then, for any two vertices $k$ and $l$ where $k \in \operatorname{chain}(a+1, i)$ and $l \in \operatorname{chain}(j, a-1)$, $E S P(k, l)$ also passes through $a$. This is a known property of the Euclidean shortest path. So, any assignment of blocking vertices to minimal invisible pairs must ensure that this property of the Euclidean shortest path between any two vertices in the corresponding polygon is preserved. Abello and Kumar suggest this property, which they call the second path-consistent assignment, as the fourth necessary condition. Assume on the contrary that there exists an assignment of blocking vertices which satisfies Necessary Condition 4 but does not satisfy the fourth necessary condition of Abello and Kumar. Using analogous arguments to those mentioned above, it can be shown that there exists an ordered cycle $C$ formed by the paths from $k$ to $l$, from $l$ to $a$, and from $a$ to $k$ such that all vertices of $C$ except $k$ and $l$ are assigned to minimal invisible pairs between the vertices of $C$, which contradicts that the assignment of blocking vertices has satisfied Necessary Condition 4 .

The above discussion shows that if any graph satisfies our four necessary conditions, then it also satisfies all necessary conditions suggested by Abello and Kumar. Since the Euclidean shortest path between any two points in a simple polygon is unique and this property can be proved from the fact that the sum of the internal angles of a simple polygon of $n$ vertices is $(n-2) 180^{\circ}$, it is natural that the second, third, and fourth necessary conditions of Abello and Kumar, which correspond to the uniqueness property of the Euclidean shortest path, follow from Necessary Condition 4.

In the same paper, Abello and Kumar have introduced a class of graphs called quasipersistent graphs and have shown that visibility graphs of simple polygons are contained in this class. They have pointed out (see Theorem 2 in [3]) that the class of quasi-persistent graphs is equivalent to the class of graphs satisfying Necessary Conditions 1 and 2. So Lemmas 3 and 4 give an alternate proof of the equivalence between quasi-persistent graphs and graphs satisfying Necessary Conditions 1 and 2. Moreover, our algorithm for testing Necessary Conditions 1 and 2 can be viewed as an algorithm for recognizing quasi-persistent graphs. In addition, the claim made by Abello et al. [1] that our four necessary conditions are not sufficient is not correct.

\section{Acknowledgments}

The author would like to thank the anonymous referees for their valuable suggestions and assistance. The author gratefully acknowledges the helpful comments and suggestions of Joseph O'Rourke, Subhash Suri, Sanjeev Saluja, Xavier Furtado, and Jaikumar Radhakrishnan in the production of this paper.

\section{References}

1. J. Abello, O. Egecioglu, and K. Kumar, Visibility graphs of staircase polygons and the weak Bruhat order, I: from visibility graphs to maximal chains, Discrete \& Computational Geometry, 14(3) (1995), 331-358. 
2. J. Abello, O. Egecioglu, and K. Kumar, Visibility graphs of staircase polygons and the weak Bruhat order, II: from maximal chains to polygons, preprint.

3. J. Abello and K. Kumar, Visibility graphs and oriented metroids, Proceeding of Graph Drawing, Lecture Notes in Computer Science, Vol. 894, Springer-Verlag, Berlin, pp. 147-158, 1995.

4. J. Abello, H. Lin, and S. Pisupati, On visibility graphs of simple polygons, Congressus Numeratium, 90 (1992), 119-128.

5. D. Avis and D. Rappaport, Computing the largest empty convex subset of a set of points, Proceedings of the First ACM Symposium on Computational Geometry, pp. 161-167, 1985.

6. M. A. Buckinghan, Circle graphs, Ph.D. Dissertation, Report No. NSO-21, Courant Institute of Mathematical Sciences, New York, 1980.

7. H. ElGindy, Hierarchical decomposition of polygons with applications, Ph.D. Dissertation, McGill University, Montreal, 1985.

8. H. Everett, Visibility graph recognition, Ph.D. Dissertation, University of Toronto, Toronto, January 1990.

9. H. Everett and D. Corneil, Recognizing visibility graphs of spiral polygons, Journal of Algorithms, 11 (1990), 1-26.

10. C. P. Gabor, W. Hsu, and K. J. Supowit, Recognizing circle graphs in polynomial time, Proceedings of the 26th IEEE Annual Symposium on Foundation of Computer Science, pp. 106-116, 1985.

11. F. Gravil, Algorithms for minimum coloring, maximum clique, minimum covering by cliques, and maximum independent set of a chordal graph, SIAM Journal on Computing, 1 (1972), 180-187.

12. S. K. Ghosh, On recognizing and characterizing visibility graphs of simple polygons, Report JHU/EECS86/14, The Johns Hopkins University, Baltimore, 1986. Also in Proceedings of the Scandinavian Workshop on Algorithm Theory, Lecture Notes in Computer Science, Vol. 318, Springer-Verlag, Berlin, pp. 96-104, 1988.

13. S. K. Ghosh, A. Maheshwari, S. P. Pal, S. Saluja, and C. E. Veni Madhavan, Characterizing and recognizing weak visibility polygons, Computational Geometry: Theory and Applications, 3 (1993), 213-233.

14. M. C. Golumbic, Algorithmic Graph Theory and Perfect Graphs, Academic Press, New York, 1980.

15. J. Hershberger, An optimal visibility graph algorithm for triangulated simple polygon, Algorithmica, 4 (1989), 141-155.

16. T. Lazano-Perez and M. A. Wesley, An algorithm for planning collision free paths among polygonal obstacles, Communications of the ACM, 22 (1979), 560-570.

17. J. O'Rourke, Ant Gallery Theorems and Algorithms, Oxford University Press, Oxford, 1987.

18. J. O'Rourke, Computational geometry column 18, SIGACT News, 24 (1993), 20-25.

19. L. G. Shapiro and R. M. Haralick, Decomposition of two-dimensional shape by graph-theoretic clustering, IEEE Transactions on Pattern Analysis and Machine Intelligence, 1 (1979), 10-19.

20. T. Shermer, Hiding people in polygons, Computing, 42 (1989), 109-132.

21. G. Srinivasaraghavan and A. Mukhopadhyay, A new necessary condition for the vertex visibility graphs of simple polygons, Discrete \& Computational Geometry, 12 (1994), 65-82.

Received October 10, 1994, and in revised form June 11, 1996. 